\title{
SHiP: a new facility with a dedicated detector to search for new neutral particles and studying tau neutrino properties
}

\author{
V.Shevchenko ${ }^{1,2, a}$, on behalf of the SHiP Collaboration. \\ ${ }^{1}$ National Research Centre "Kurchatov Institute" ac.Kurchatova sq., 1, Moscow 123182 Russia \\ ${ }^{2}$ Far Eastern Federal University, Sukhanova str. 8, Vladivostok 690950 Russia
}

\begin{abstract}
.
SHiP (Search for Hidden Particles) is a new general purpose fixed target facility, whose Technical Proposal has been recently reviewed by the CERN SPS Committee and by the CERN Research Board. The two boards recommended that the experiment proceeds further to a Comprehensive Design phase in the context of the new CERN Working group "Physics Beyond Colliders", aiming at presenting a CERN strategy for the European Strategy meeting of 2019. In the initial phase of SHiP, the $400 \mathrm{GeV}$ proton beam extracted from the SPS will be dumped on a heavy target with the aim of integrating $2 \times 10^{20}$ pot in 5 years. A dedicated detector, based on a long vacuum tank followed by a spectrometer and particle identification detectors, will allow probing a variety of models with light long-lived exotic particles and masses below $O(10) \mathrm{GeV} / \mathrm{c}^{2}$. The main focus will be the physics of the so-called Hidden Portals, i.e. search for Dark Photons, Light scalars and pseudo-scalars, and Heavy Neutrinos. The sensitivity to Heavy Neutrinos will allow for the first time to probe, in the mass range between the kaon and the charm meson mass, a coupling range for which Baryogenesis and active neutrino masses could also be explained. Another dedicated detector will allow the study of neutrino cross-sections and angular distributions.
\end{abstract}

\section{Introduction}

There had been many expectations before LHC started, notably Higgs boson or strong interaction of vector bosons («guaranteed discovery») and possible New Physics phenomena in TeV ballpark (SUSY particles? extra dimensions? compositeness? etc). After five years the situation can be characterized as the Standard Model impressive triumph: the Higgs boson is discovered, many tiny and highly nontrivial SM effects in flavor physics and rare decays are confirmed, and nothing is found beyond SM. On the other hand, we still know (not from LHC) that there is physics beyond the SM: neutrino masses and oscillations; dark matter; baryon asymmetry of the Universe show that clearly. Besides those observational facts, there are many «why» and «how» in the SM itself: how is EW scale so smaller than UV scale? why hierarchy between SM scales? why are lefts doublets and rights singlets? why 3 generations? why CKM hierarchy? why CP? etc. Speaking in more theoretical prospective one can ask, is the SM a consistent field theory in the first place? Generally

a e-mail: Vladimir.Shevchenko@ cern.ch 
speaking, the answer to this question is negative. Existence of Landau pole, anomalies, naturalness issues precludes one to consider SM as fully satisfactory theoretical framework. On the other hand, it is also a fact of life that the things in the SM are arranged in such a tricky way, that (almost) all the above mentioned problems are either cured or seem not to be physically relevant. Nice example is anomaly cancellation between quark and lepton sectors, which would not be possible without delicate SM charge distribution between particles.

Let us remind that there are many various scales and small numbers in the SM with intricate relations between them. They include

- dimensionless gauge coupling constants: $\alpha_{e m}=(137.035999679)^{-1} ; \alpha_{s}\left(m_{Z}\right)=(8.503)^{-1}$

- Yukawa constants: $m_{u, d} / \Lambda=(0.5 \div 2) \% ; \Lambda / m_{b}=(6 \div 24) \% ; m_{e} / m_{t}=3 \cdot 10^{-6}$

- QCD to EW scale ratio: $G_{F} m_{p}^{2}=10^{-5}$

- CKM matrix parameters: $\lambda=\left|V_{u s}\right|=0.22$

There are even more mysterious relations like Koide relation between lepton masses or ZeldovichBjorken relation between QCD and cosmological scales. Another instructive example is UVregularization, corresponding to inclusion of some (artificial) information about theory behavior in ultraviolet region. In renormalizable theories all physics above $\Lambda$ can be encoded just in a couple of numbers - coefficients in front of marginal operators like the famous fine structure constant $1 / 137$ in QED. Renormalizability, however, does not mean naturalness. Computing, for example, average of some local $d$-dimensional operator $\mathcal{T}[\phi]$ in a theory with UV cutoff $\Lambda$, one gets typically (up to, perhaps, logarithms):

$$
\int \mathcal{D} \phi \mathcal{T}[\phi] e^{i S[\phi]} \sim c \cdot \Lambda^{d}+\text { finite part }
$$

The first term characterizes ad hoc assumptions about the integration measure/detector (for example, geometry of the lattice with the link size $a \sim 1 / \Lambda$ used for computation), but not the genuine physics of this operator. Of course in many cases symmetries of the theory guarantee $c=0$, but if not, we have to work out a way of disentangling "the physics of the detector" from "the physics of the physics" (which presumably is hidden in the finite part). Notable examples include vacuum energy density, Higgs boson mass and gluon condensate, where only in the latter case the answer is known (qualitatively, but not quantitatively) - quantum scale anomaly of Yang-Mills theory and dimensional transmutation take care of unphysical UV scale $\Lambda$ to become physical scale $\Lambda_{Q C D}$. In other cases of this sort, like cosmological constant problem or hierarchy problem a solution is yet to be found.

Perhaps the most important qualitative result of LHC is experimental observation that the observable Higgs mass corresponds to metastability of the SM vacuum (see Fig.1).

Incomplete list of researchers who contributed to this issue includes I.V. Krive, A.D.Linde, N.Cabibbo, L.Maiani, G.Parisi, R.Petronzio, M.Lindner, H.B.Nielsen, C.Froggatt, J. Elias-Miro, J. R. Espinosa, G. F. Giudice, G. Isidori, A. Riotto, A. Strumia, J. R. Espinosa, M. Quiros, G.Altarelli. This result gives a strong hint that there is no heavy New Physics degrees of freedom up to the Planck scale. It is worth reminding that historically the top and Higgs masses were predicted [1] from criticality to be $173 \pm 5 \mathrm{GeV}$ and $135 \pm 9 \mathrm{GeV}$ back to 1995 .

If indeed no NP degrees of freedom can be found at energy frontier it is perhaps more useful to think not about NP that could solve theoretical problems of the SM, but about NP that could explain observed effects beyond SM. It is remarkable that such scenarios could have NP degrees of freedom as relatively light (a few GeV) but very weekly interacting SM singlets (see Fig.2). It is clear that there are two crucial requirements one has to obey for experimental search of such particles: huge initial intensity and large (to be more precise, very long) controlled detector volume. A project of experimental facility capable to do the job is described below. 


\section{Motivations for the SHiP Project}

SHiP (Search for Hidden Particles) [3, 4] is a new general purpose fixed target facility proposed at the CERN SPS accelerator to search for particles predicted by Hidden Portals. These particles are expected to be predominantly accessible through the decays of heavy hadrons. The facility is therefore designed to maximise the production and detector acceptance of charm and beauty mesons, while providing the cleanest possible environment. The $400 \mathrm{GeV}$ proton beam extracted from the SPS will be dumped on a high density target with the aim of accumulating $2 \times 10^{20}$ protons on target during 5 years of operation. The charm production at SHiP exceeds any existing and planned facility. SHiP facility is supposed to be located in the North Area and shares TT20 transfer line and the slow extraction mode with other fixed target programmes.

A general layout is depicted on Fig.3. Water cooled target will dissipate $4 \times 10^{13}$ protons at 7 sec which gives average power $355 \mathrm{~kW}$ (2.56 MW during $1 \mathrm{sec}$ spill). Initial dose is estimated to be around $50 \mathrm{~Sv} / \mathrm{h}$. The target is segmented: high-Z hybrid solution is composed of molybdenum alloy strips and pure tungsten ones.

The main decay vessel (in vacuum option) has the size $10 \times 5 \times 60$ meters. Liquid or plastic scintillator options for veto system (with the readout by optical modules) are being considered.

A dedicated detector, based on a long vacuum tank followed by a spectrometer and particle identification detectors, will allow probing a variety of models with light long-lived exotic particles and masses below $O(10) \mathrm{GeV} / \mathrm{c}^{2}$. Since the hidden sector particles originating from charm and beauty are produced with a significant transverse momentum with respect to the beam axis, the detector should be placed as close as possible to the target. A critical component of SHiP is the muon shield, which deflects the high flux of muons produced in the target, that would represent a very serious background for the particle searches, away from the detector. The detector is designed to fully reconstruct the exclusive decays of hidden particles and to reject the background down to below 0.1 events in the sample of $2 \times 10^{20}$ protons on target. The spectrometer is designed to accurately reconstruct the decay vertex, mass and impact parameter of the decaying particle at the target. A set of calorimeters followed by muon chambers provide identification of electrons, photons, muons and charged hadrons. A dedicated timing detector measures the coincidence of the decay products, which allows the rejection of combinatorial backgrounds. The decay volume is surrounded by background taggers to tag neutrino and muon inelastic scattering in the surrounding structures, which may produce long-lived SM particles, such as $\mathrm{K}_{\mathrm{L}}$, that have similar topologies to the expected signals.

From theoretical point of view it is useful to discuss search for Hidden Particles in terms of portals. The total Lagrangian has the following form:

$$
L=L_{S M}+L_{H S}+L_{\text {Portal }}
$$

where Hidden Sector (HS) contains Dark Matter and can have very complicated structure, while $L_{\text {Portal }}$ contains terms responsible for interaction between HS particles and some singlet operator built from SM fields. People analyse renormalizable portals (scalar, vector and neutrino) as well as higher dimensional ones (see references in [4]). Of particular interest is the so called $v$ MSM model [5], with the Largangian

$$
L=L_{S M}+\bar{N}_{I} i \partial_{\mu} \gamma^{\mu} N_{I}-F_{\alpha I} \Phi \bar{N}_{I} L_{\alpha}-\frac{M_{I}}{2} \bar{N}_{I}^{c} N_{I}+\text { h.c. }
$$

The new particles - Heavy Neutral Leptons - are schematically depicted on Fig.4. Particle $N_{1}$ with mass in $\mathrm{keV}$ range plays a role of dark matter, while $N_{2}$ and $N_{3}$ with $\mathrm{GeV}$-scale masses are responsible for the SM neutrino masses via see-saw mechanism and also for baryogenesis. These particles are among the objects to search for at the SHiP facility. 
Let us estimate the number of Hidden Sector events. In general it is given by

$$
n(H S)=N(\text { pot }) \times \chi(p p \rightarrow H S) \times \mathcal{P}_{v t x} \times \mathcal{A}_{t o t}(H S \rightarrow \text { visible })
$$

where $N(p o t)=2 \times 10^{20}$ and $\mathcal{P}_{v t x}$ is probability that Heavy Neutral Lepton (HNL) of a given mass and couplings decays in the SHiP fiducial volume. One gets for the detector acceptance for all $\mathrm{HNL}$ final (visible) states

$$
\mathcal{A}_{t o t}(H S \rightarrow \text { visible })=\sum_{i} \operatorname{Br}(H N L \rightarrow i) \times \mathcal{A}(i)
$$

with

$$
\chi(p p \rightarrow H N L)=2 \sum_{q=c, b} \chi(p p \rightarrow q \bar{q}) \times \operatorname{Br}(q \rightarrow H N L) \times U^{2}
$$

where mixing matrix $U^{2}=U_{e}^{2}+U_{\mu}^{2}+U_{\tau}^{2}$ and conservative numerical estimates give $\mathcal{P}_{v t x} \times \mathcal{A} \times$ Selection $\sim 10^{-6}$, with $\chi(p p \rightarrow c \bar{c})=1.7 \times 10^{-3}, \chi(p p \rightarrow b \bar{b})=1.6 \times 10^{-7}$ for $400 \mathrm{GeV}$ protons on Molybdenum target. This shows crucial importance of initial multiplicity. Typical SHiP sensitivity plots from [3, 4] are depicted on Fig.5 and Fig.6.

The experimental facility is also ideally suited for studying interactions of tau neutrinos. It will therefore host an emulsion cloud chamber based on the OPERA detector concept, upstream of the hidden-particle decay volume, followed by a muon spectrometer. For the total number of neutrino one can estimate

$$
N_{v_{\tau}+\bar{v}_{\tau}}=4 N_{p o t} \frac{\sigma_{c \bar{c}}}{\sigma_{p N}} f_{D_{s}} \operatorname{Br}\left(D_{s} \rightarrow \tau\right)=2.85 \times 10^{-5} N_{p o t}
$$

The resulting expected multiplicity factors in SHiP in comparison with DONUT and CHARM experiments for tau-neutrino and Heavy Neutral Leptons are

$$
\frac{N\left(v_{\tau}\right) @ S H i P}{N\left(v_{\tau}\right) @ D O N U T} \sim 600 \text { and } \frac{N(H N L) @ S H i P}{N(H N L) @ C H A R M} \sim 10000
$$

\section{Conclusions}

It had been customary to argue, before LHC started, that hierarchy problem pointed to a few TeV scale as a reasonable one for New Physics (NP). Supersymmetric extension and other scenarios beyond the Standard Model had been intensively studied. In reality no these new particles have been discovered by ATLAS and CMS experiments (leaving aside the $126 \mathrm{GeV}$ Higgs boson). Moreover, indirect searches of NP by LHCb and other precision experiments provided very high, hundreds-TeV bounds for NP degrees of freedom in some channels (without special assumptions about flavor alignment). On the other hand the experimentally known New Physics phenomena (neutrino oscillations, dark matter etc) give no indication to the intrinsic scales of the corresponding physics. From theoretical side, all that calls for reconsideration of the Standard Model as being the theory probably adequately describing physics up to the Planck scale. From experimental point of view, this can be interpreted as an argument in favor of NP (responsible, in particular, for neutrino oscillations and dark matter) manifested by relatively light, but very weekly interacting particles. The search of the latter should be done on intensity frontier rather than on energy frontier, and the proposed Search for Hidden Particles (SHiP) experiment at CERN's SPS, discussed in the present talk, is an example of this kind. Search for Hidden Particles (SHiP) experiment is complement to searches for New Physics at energy frontier at CERN. It is proposed to search for New Physics in the largely unexplored domain of new, very weakly interacting particles with masses $O(10) \mathrm{GeV} / \mathrm{c}^{2}$. It provides unique opportunities for tauneutrino studies. Sensitivity improves previous experiments by factor $O\left(10^{4}\right)$ for Hidden Sector and by $O\left(10^{2}\right)$ for neutrino physics. The impact of the discovery of new light hidden particles is hard to overestimate. 


\section{References}

[1] C. D. Froggatt and H. B. Nielsen, Standard model criticality prediction: Top mass 173 +5-GeV and Higgs mass 135 +- 9-GeV, Phys. Lett. B 368 (1996) 96, hep-ph/9511371.

[2] D. Buttazzo, G. Degrassi, P. P. Giardino, G. F. Giudice, F. Sala, A. Salvio and A. Strumia, JHEP 1312 (2013) 089, arXiv:1307.3536.

[3] The SHiP Collaboration, A facility to Search for Hidden Particles (SHIP) at the CERN SPS, April 2015, arXiv:1504.04956.

[4] S. Alekhin et al., A facility to Search for Hidden Particles at the CERN SPS: the SHiP physics case, Rept. Prog. Phys. 79 (2016) no.12, 124201, arXiv:1504.04855 [hep-ph].

[5] T. Asaka, S. Blanchet and M. Shaposhnikov, The nuMSM, dark matter and neutrino masses, Phys. Lett. B 631 (2005) 151, hep-ph/0503065. 

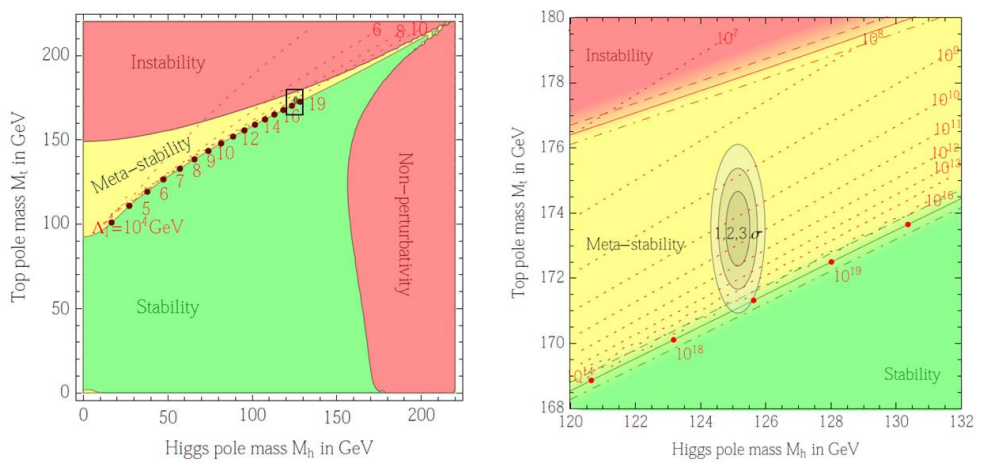

Figure 1. (Meta)-Stability of the SM vacuum (from [2]).

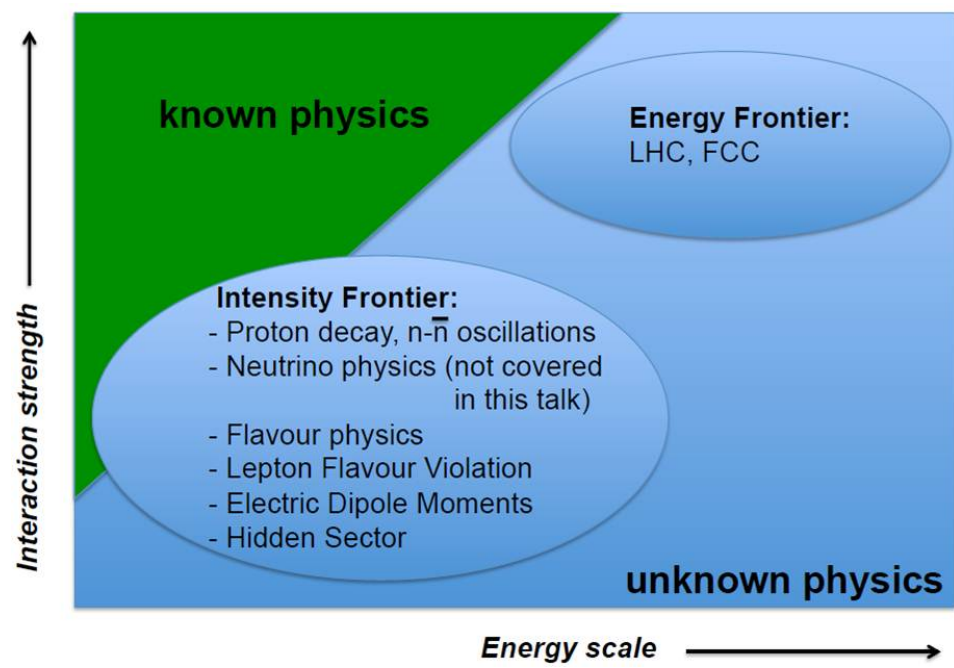

Figure 2. Energy Frontier vs Intensity Frontier 


\section{The SHiP experiment at SPS}

(as implemented in Geant4)

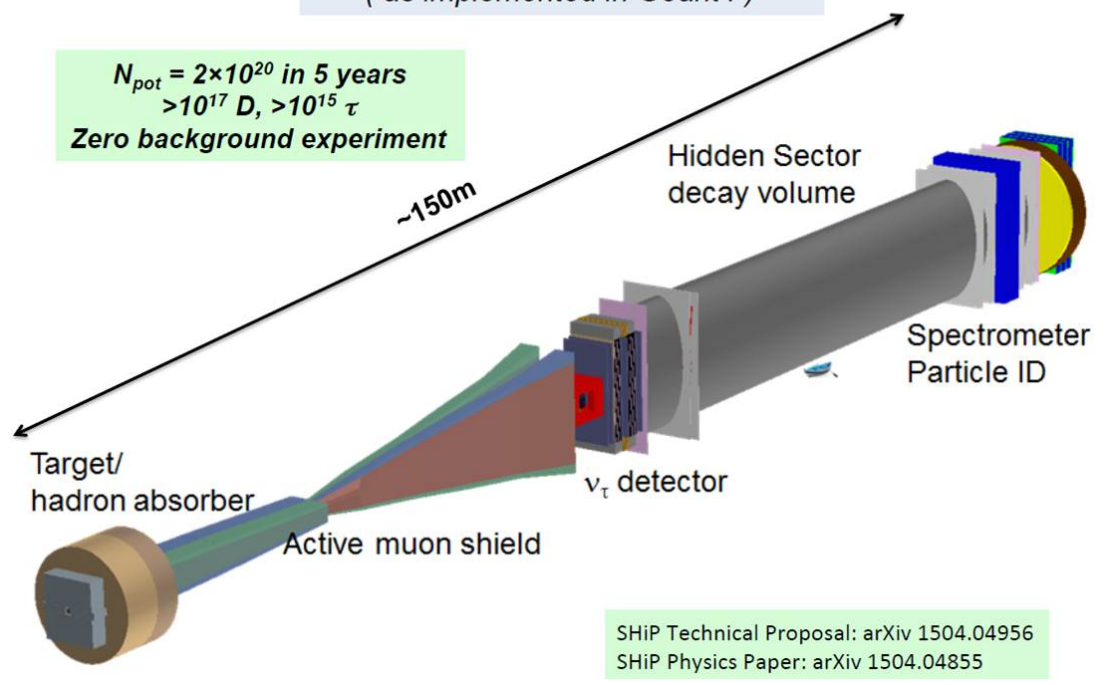

Figure 3. General Layout of SHiP experiment
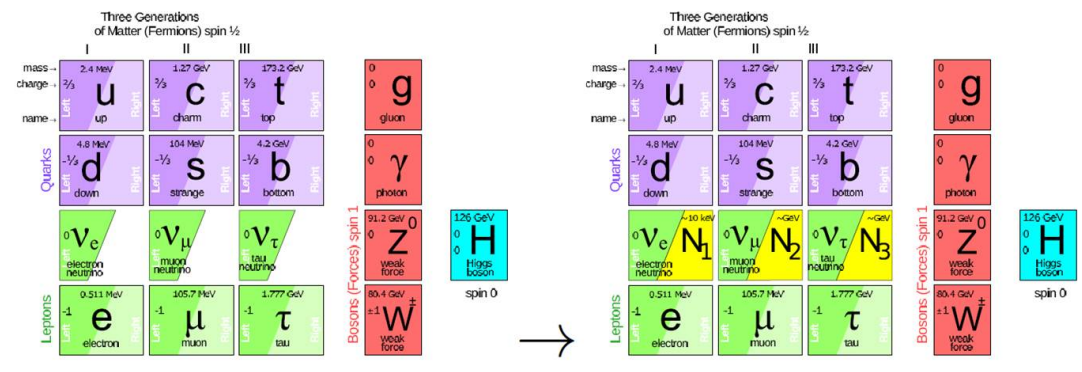

Figure 4. nu-Minimal Standard Model 


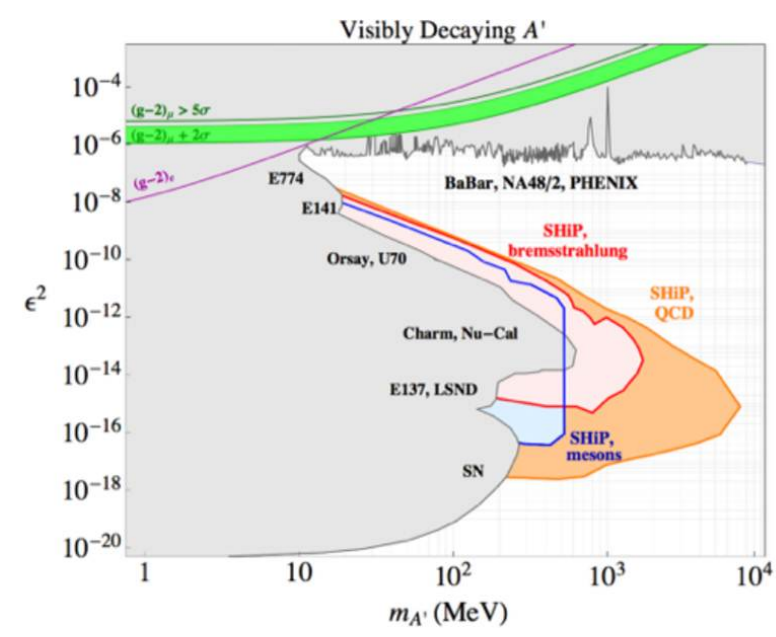

Figure 5. SHiP sensitivity to dark photons
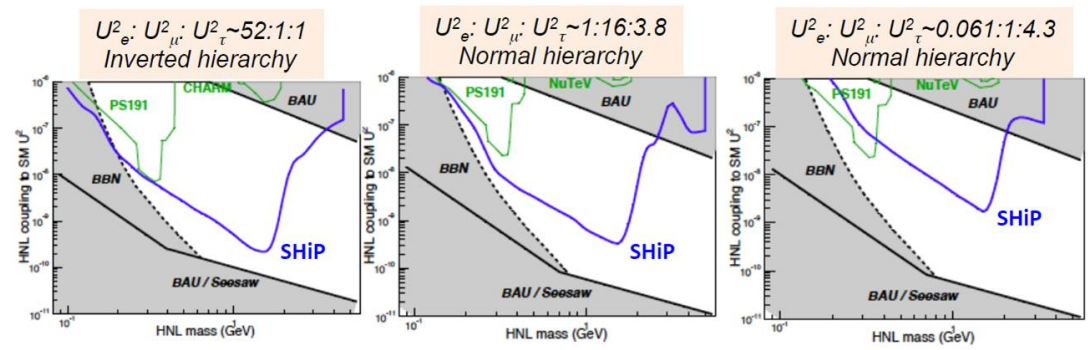

Figure 6. SHiP sensitivity to $\mathrm{HNL}$ 